\title{
Model of Anisotropic Electrical Resistivity in Rough Thin Films
}

\author{
T. Szumiata And M. Gzik-Szumiata \\ Department of Physics, Technical University of Radom, I. Krasickiego 54, 26-600 Radom, Poland \\ In this work a new model of electrical resistivity is proposed in order to study the relationship between surface \\ roughness geometry and thin films resistivity. The model is based on the numerical dynamic averaging of electron \\ mean free path over whole simulated structure of rough film. For current-in-plane configuration the resistivity \\ increases with decreasing film thickness faster than for current-perpendicular-to-plane one. Our simulations \\ showed that big roughness depth and fine in-plane spatial period of roughness are crucial factors increasing the \\ resistivity of ultrathin metallic layers.
}

PACS numbers: 73.50.-h, 73.61.At, 73.23.Ad, 75.70.Ak, 75.47.-m

\section{Introduction}

Fuchs and Sondheimer theory $[1,2]$ of conduction electrons diffusive scattering at the film surface underestimates the resistivity for ultrathin layers because it does not take into account a surface roughness. Surface roughness influences not only electrical conductivity but also other physical properties e.g. surface magnetostriction [3]. In this work a new model of electrical resistivity is developed in order to predict the dependence of thin films resistivity on geometrical parameters of surface roughness. Especially important aspect of this investigation is the anisotropy of the resistivity i.e. difference between resistivity for current-in-plane (CIP) and current-perpendicular-to-plane (CPP) configurations caused by superficial roughness. Though the model does not consider magnetism it could be useful in precise analysis of giant magnetoresistance (GMR) and spin transfer torque (STT) experimental data for magnetic multilayers.

\section{Model}

The structure of thin single film (of thickness $t$ ) with two rough surfaces has been generated numerically in 2-dimensional approximation (see insets in Fig. 1). The roughness has been characterized by two parameters: depth $-\sigma$, and half of space period $-\xi$. Direct proportionality of the electrical conductivity to mean free path (MFP) $\left\langle L_{0}\right\rangle$ has been assumed (i.e. the resistivity $\rho$ is inversely proportional to $\left\langle L_{0}\right\rangle$ ).

The movement of electrons (in one-electron picture) is simulated dynamically over whole rough thin layer. If electron does not meet any obstacle its local mean free path is equal to MFP $L_{0}$ for bulk material. However, when it collides with the boundaries of the structure the MFP shortens. After scattering the electron changes ran- domly its direction of movement. For CIP configuration electron starts from left side at mid-height position. When electron reaches right side of the structure it disappears and new electron occurs at starting point.

The final value of MFP (i.e. $\left\langle L_{0}\right\rangle$ ) is obtained by averaging over whole electron trajectory after 10000 collisions. For CPP configuration electron starts from the centre of top surface and finishes its movement at bottom surface, however this final collision is not classified as diffusive scattering but rather as the end of ballistic transport process. In this case it is assumed for simplicity that a local free path takes a maximal value i.e. $L_{0}$.

\section{Numerical results}

A natural consequence of the proposed model is the anisotropy of the resistivity for CIP and CPP configurations, which is illustrated in Fig. 1. When thickness $t$ of the film is comparable or smaller than bulk free path $L_{0}$ the CIP resistivity increases much faster than CPP one. The results of simulations presented in Fig. 1a-d convinces that big roughness depth and fine in-plane spatial period of roughness are crucial factors increasing the resistivity of ultrathin metallic layers. One should underline that CIP resistivity is significantly more sensitive to surface roughness.

\section{Discussion}

Present results concerning thickness dependence of CIP resistivity for smooth films are very close to those in former work [4] obtained by static averaging of isotropic MFP. However in the case of rough surfaces the present model predicts higher CIP resistivity by about $20 \%$ for $t / L_{0} \approx 0.5$ and by about $40 \%$ for $t / L_{0} \approx 0.25$. It could be explained by the fact that new model based on dynamic simulation considers in the natural way a trapping 
of electrons by surface roughness. Another advantage of our dynamical simulation is not utilizing phenomenological Matthiesen's rule for effective mean free path - in contrary to very recent analytical model described in paper [5] (containing a detailed review of old models $[1,2,6]$ and relating electrical conductivity to thermal conductivity). Moreover none of other models described in the listed literature predicts CIP-CPP anisotropy of electrical resistivity for thin films.
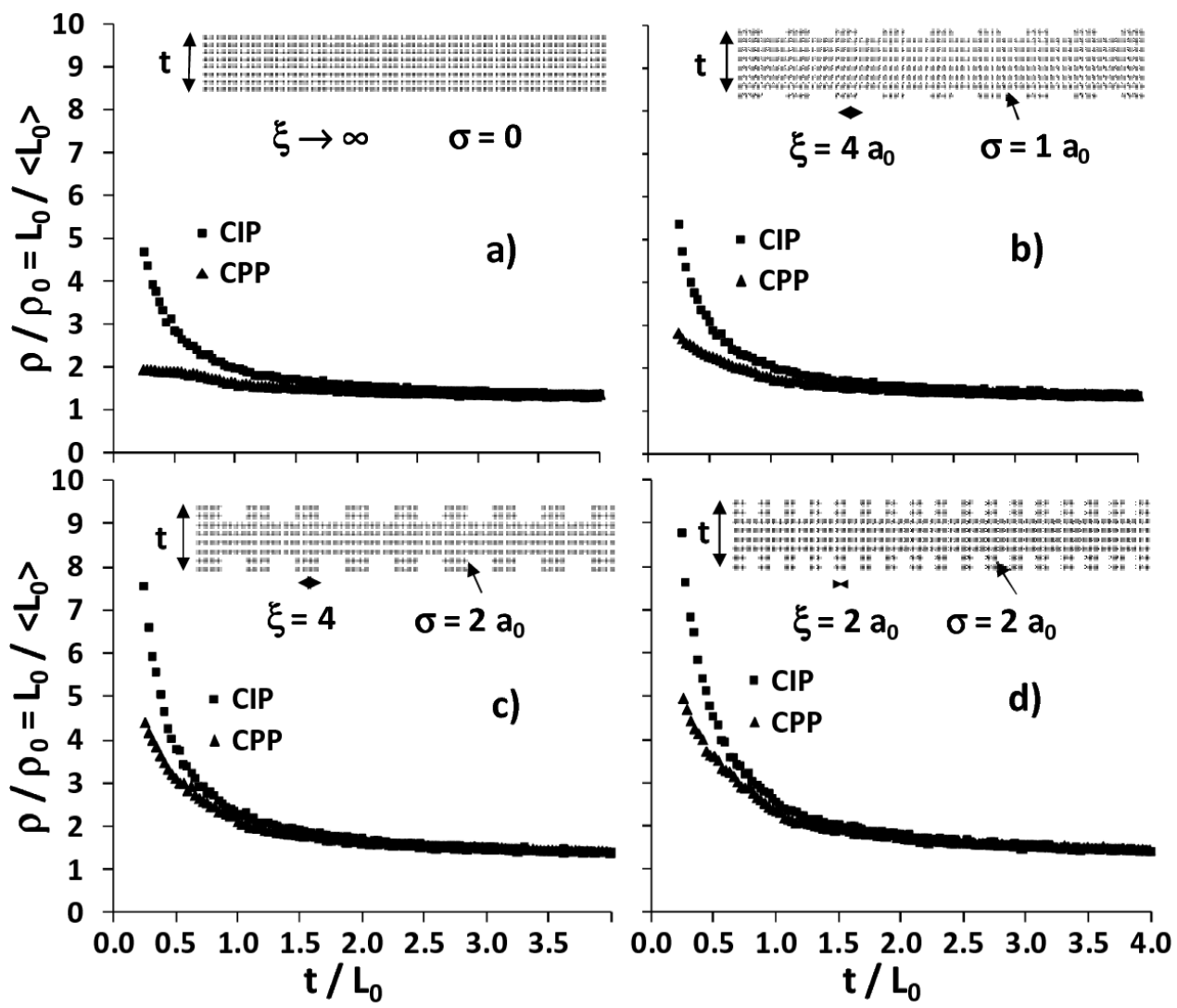

Fig. 1. Roughness and thickness dependence of relative CIP and CPP resistivity.

\section{Conclusions}

Dynamic simulation of electron movement through rough thin film structure enables to distinguish CIP and CPP configurations in resistivity estimation. Presented model points to the roughness depth and in-plane spatial period of roughness as very important parameters materially affecting electrical resistivity of thin films. We plan to extend the model in order to consider the influence of grain boundaries on mean free path in polycrystalline metallic films (like in [5] for bulk polycrystalline alloys). We also suggest a future development of the model by transition from 2D to 3D geometry of surface roughness as well as a consideration of more realistic description of ballistic transport in CPP configuration.

\section{References}

[1] K. Fuchs, Proc. Cambridge Philos. Soc. 34, 100 (1938).

[2] E.H. Sondheimer, Adv. Phys. 1, 1 (1952).

[3] T. Szumiata, M. Gzik-Szumiata, K. Brzózka, Mater. Sci. Poland 26, 1039 (2008).

[4] T. Szumiata, M. Gzik-Szumiata, K. Brzózka, Acta Phys. Superfic. 11, 126 (2009).

[5] J.S. Jin, J.S. Lee, O. Kwon, Appl. Phys. Lett. 92, 171910 (2008).

[6] A.F. Mayadas, M. Shatzkes, Phys. Rev. B 1, 1382 (1970). 